\title{
The constant temperature control system design based on STM32 and PID algorithm
}

\author{
Zhao Xuyang ${ }^{1, ~ a ~ a n d ~ Y a n g ~} \mathrm{Hao}^{2, \mathrm{~b}}$ \\ ${ }^{1}$ School of Zhao Xuyang, China Jiliang University, Hangzhou 310000, China \\ ${ }^{2}$ School of Yang Hao, China Jiliang University, Hangzhou 310000,China \\ azhaoxuyangmail126.com, ,yanghao@cjlu.edu.cn
}

Keywords: PID Algorithm; STM32; semiconductor temperature regulator; constant temperature

\begin{abstract}
Time-varying, nonlinear and multivariable coupling are characteristics of temperature. In the temperature control process, the detected temperature is often lags behind the regulation of temperature, which will cause the phenomenon such as the temperature of the controlling system overshoot and temperature oscillation. Temperature control is proposed based on incremental PID algorithm model in this paper, the system uses low-power STM32 as the main chip, DS18B20 digital temperature sensor and semiconductor temperature regulator. Experimental results show that the system can effectively maintain the temperature of the system constant.
\end{abstract}

\section{Introduction}

In natural environment, the system will generate a heat exchange which is difficult to control with the outside world, and produce unpredictable interference.During this situation it will be difficult to achieve in precise temperature controlling. When performing high-precision temperature control, temperature tends to produce overshoot phenomenon [1].

Temperature control system with a lag, nonlinear and time-varying characteristics, can not establish a precise mathematical model, therefore, the use of conventional linear control theory can not achieve satisfactory control effect. semiconductor temperature controller working condition have a relationship with heat conditions and the environment factors. its work process is a non-steady state process, it needs to be addressed precisely controlled .PID control theory has a characteristic of convenient parameter setting, flexible structural change, robust and easy to implement [1,2]. the system design using incremental PID algorithm can not only solve the above problems, but also in the unsupervised, for a long time temperature will be automatically collected, automatic controlling of the semiconductor temperature controller achieves heating or cooling function, the system temperature will always be maintained constantly.

Using a master chip STM32 and digital temperature sensor DS18B20 design a constant temperature system, the advantages consist of anti-interference digital signal, high sensitivity, fast response, and reasonable controlling of semiconductor temperature regulator,through the whole system design can effectively realize a special case temperature stable. within the setting temperature model of a small area in the design of a system with the column a special case. the result shows that the temperature controlling system of constant small area with very good results after analysis.

\section{System hardware design}

\section{Hardware System features modular design}

DS18B20 digital temperature sensor is placed on both sides of a special case, the datas are directly send to the master chip STM32 so that microchip could obtain temperature value, According to the requirements of the system setting temperature judgment mainly adopts the cooling method in the operation of the semiconductor temperature regulator in regulating or adjust temperature with heating methods . adjusting the way through the PID control algorithm of the 
temperature read by the line processing, while the master semiconductor chip STM32 control thermostat-off, so that a special case temperature maintained at a stetted temperature. this system without manual monitoring, and data can be collected via the RS232 serial port and then the observed system temperature curve plotted, by autonomous control system effectively maintained a constant ambient temperature for a long time. Figure 1 is a system hardware long block diagram.

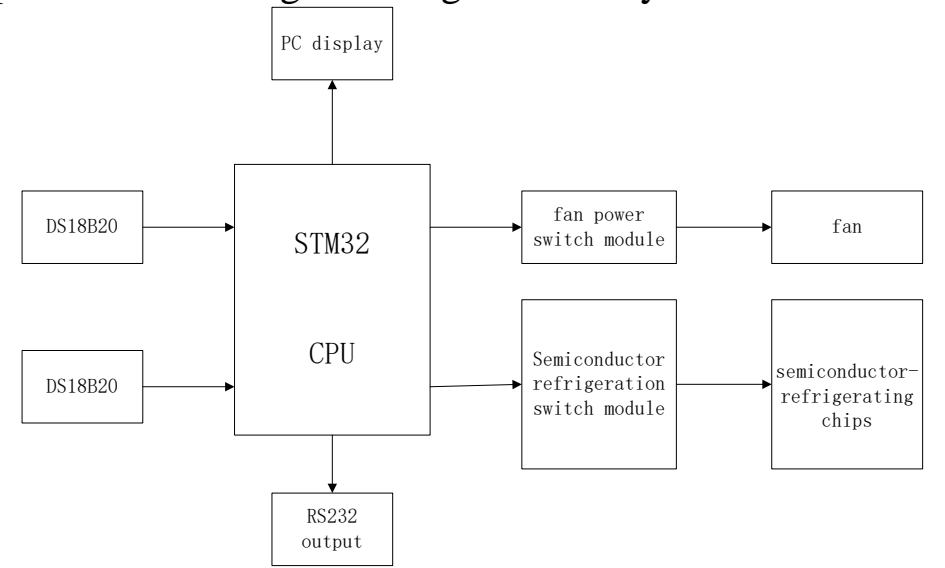

Figure 1 Diagram of system hardware block

The hardware system module functional design

System functional hardware modules include temperature acquisition module, data display and export module, fan power switch module, semiconductor temperature controller module five modules.

\section{Temperature acquisition module}

Temperature acquisition module uses a digital temperature sensor DS18B20, the sensor has high measurement accuracy, the output signal is digital with anti-jamming performance, no front-end data processing module, direct access to the STM32's I / O port , the master chip can directly read data.

\section{Data display and export module}

Taking the versatility of the system into account, used in the design is one of the communication interfaces RS232 computer data communication channel, data communication is actually using a USB data format. In this communication stepper can get higher data transfer speeds, true plug and play, it can also make it easy to connect the communication between different devices.

When data is displayed using USB to serial cable to the PC, using serial debugging assistant can easily read the temperature data acquisition, and also can import the data into a computer terminal for data storage. Therefore, the use of a standard interface technology can effectively solve the problem of inconsistent communication protocols [4].

\section{Fan power switch module}

When the semiconductor thermostat is in the cooling operation state, it is important to timely dissipate the heat, otherwise it will make semiconductor refrigeration unnormal. According to the determination of the fan work condition, by the cathode of high and low level control fan switch, the anode is normal power supply connection. This module is controlled by the master chip I / O port output level to control the fan switch purposes.

\section{Semiconductor temperature regulator module}

The core of the temperature control system is semiconductor temperature regulator. The semiconductor temperature regulator reliability is relatively high, while the power supply terminal through the access of different polarity power supply, it can absorb heat and release heat so as to achieve the effect of refrigeration and heating. using this module is characterized by the use of a device can replace separate heating and cooling systems. precise temperature control thermostat semiconductor characteristics to facilitate the composition of automated control systems [1,2]. Figure 2 is a semiconductor temperature control circuit diagram of a switching regulator. 


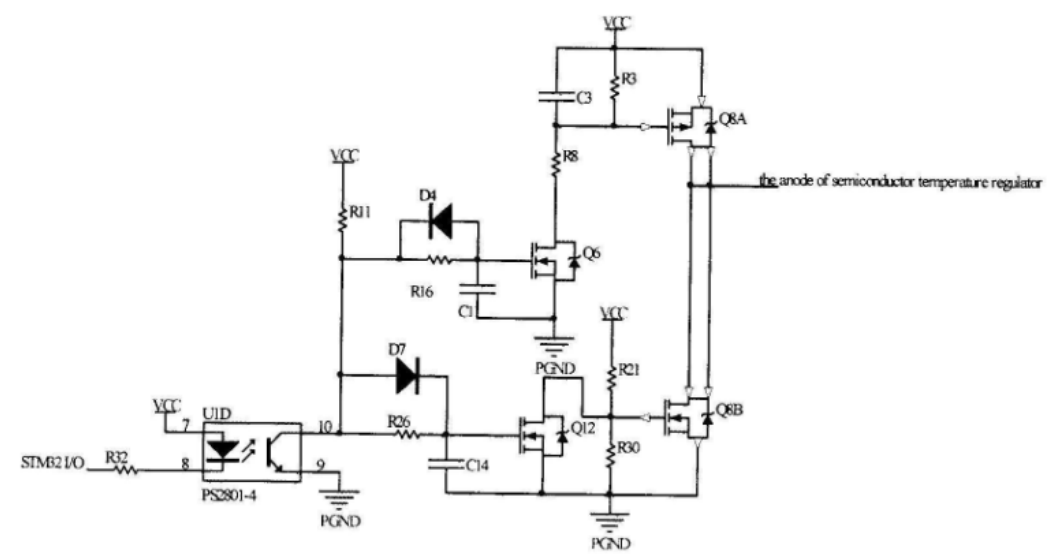

Figure 2 Diagram of semiconductor thermostat switch control circuit

\section{Software system and algorithm design}

The system software design process includes temperature digital signal acquisition, temperature display 、 PID algorithm temperature control 、 temperature feedback components. The main part of the PID control algorithm is changing the value of the ambient temperature and after the feedback the temperature regulation value, it ultimately achieve the effect of Steady-State accuracy.

\section{PID algorithm design}

PID algorithm has a simple structure, the robust performance is good, high reliability, easy parameter setting features .P, I, D control law have their own separate areas, performing linear combination constitutes control amount, then the control amount will control objects [5,6].

In the control system, a system based on real-time temperature and the set threshold value increment controls semiconductor temperature regulator operation. Therefore, the output portion of the controller is required to control the amount of incremental, in the design of the system is used incremental PID algorithm [7,8]. equation for the incremental PID algorithm is as follows.

$\triangle \mathrm{u}=\mathrm{A} \cdot \mathrm{e}(\mathrm{k})+\mathrm{B} \cdot \mathrm{e}(\mathrm{k}-1)+\mathrm{C} \cdot \mathrm{e}(\mathrm{k}-2)$

Where: $\triangle \mathrm{u}$ increment control quantity; ratio of $\mathrm{A}, \mathrm{B}, \mathrm{C}$ as PID control, differentiation, integration coefficients; e $(\mathrm{k})$, e $(\mathrm{k}-1)$, e $(\mathrm{k}-2)$ before and after the three measurements the temperature difference .

Precision of the digital temperature sensor DS18B20 can reach $\pm 0.5{ }^{\circ} \mathrm{C}$, when setting the thermostat system temperature threshold, typically the change of temperature thermostat system is set within $\pm 0.5{ }^{\circ} \mathrm{C}$, partly because the system itself and the temperature sensor error performance limits; on the other hand with a time-varying temperature, constant temperature control need to constantly switch control semiconductor thermostat and fan control to a large extent, this will reduce the life of the instrument, and even burn the instrument.

The main process of PID algorithm controller is parameter tuning, tuning in essence is through changing the regulator parameters to match the characteristics of properties and processes in order to improve the dynamic and static index system, so as to achieve the best control effect parameters. in tuning process, the first controller is as a pure proportional controller, form a closed loop, changing the coefficients, so that the coefficient corresponding to the input reaches a critical state (oscillation amplitude). Last in turn introduced differential and integral parameters according to attenuation 1 / 4 obtained, this attenuation can take into account the stability and rapidity.

\section{The result of the experiment and analysis}

Real entire test system shown in Figure 3, including the serial communication section, column oven, control panel and temperature control systems DS18B20, fans, and other semiconductor temperature regulator. 


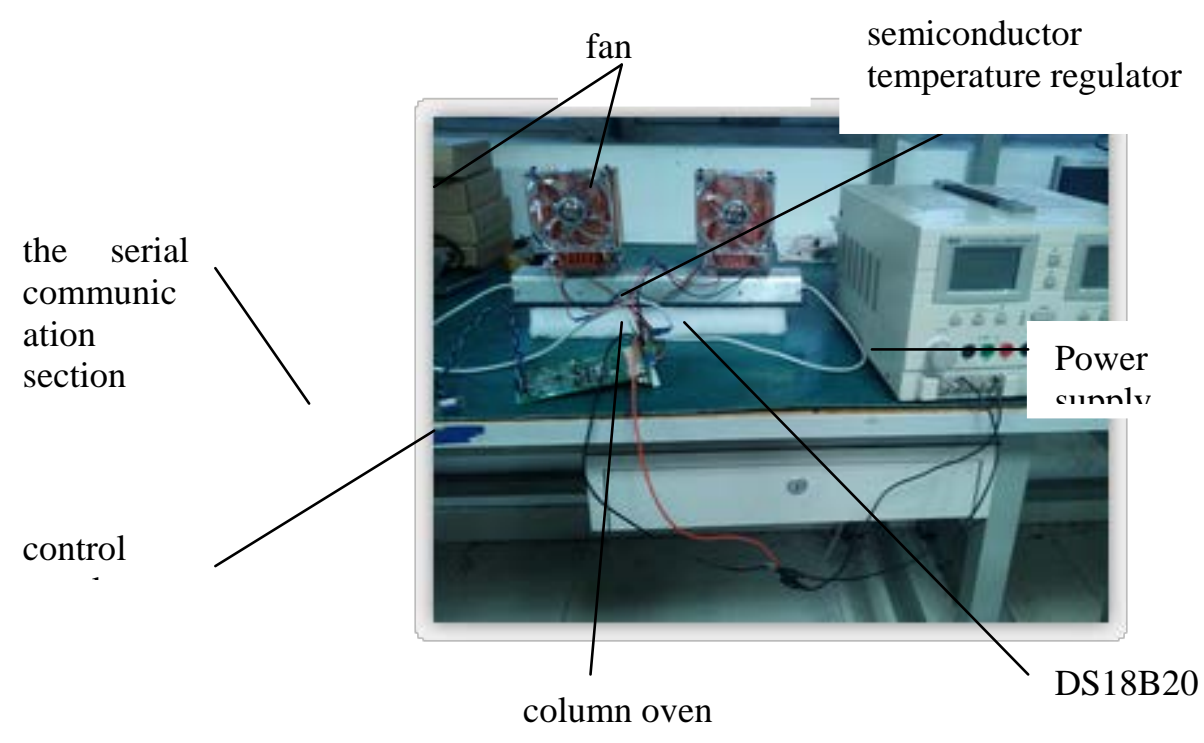

Figure 3 System schematic diagram

First obtaining room temperature, setting the thermostat system defined temperature less than room temperature, then connecting to the PC serial display interface, running the system, it will be observed that the fan is running, the positive power semiconductor thermostat is in cooling state, when the temperature is close to the serial display system the lower limit set temperature, the fan and the temperature of the semiconductor regulators are turned off, followed by heat exchange with the outside of the system will cause the temperature to rise, when the temperature rises to the set temperature limit, the fan is turning and positive power semiconductor in the temperature regulator cooling state, repeating the cooling state maintains the temperature at the set range. Column Compartment closed box is a small area, gathering room temperature is $25.4{ }^{\circ} \mathrm{C}$, the set temperature for the system is $20{ }^{\circ} \mathrm{C}$, when the permissible error set upper and lower threshold values were $20.5{ }^{\circ} \mathrm{C}$ and $19.5{ }^{\circ} \mathrm{C}$, the temperature control results shown in Figure 4

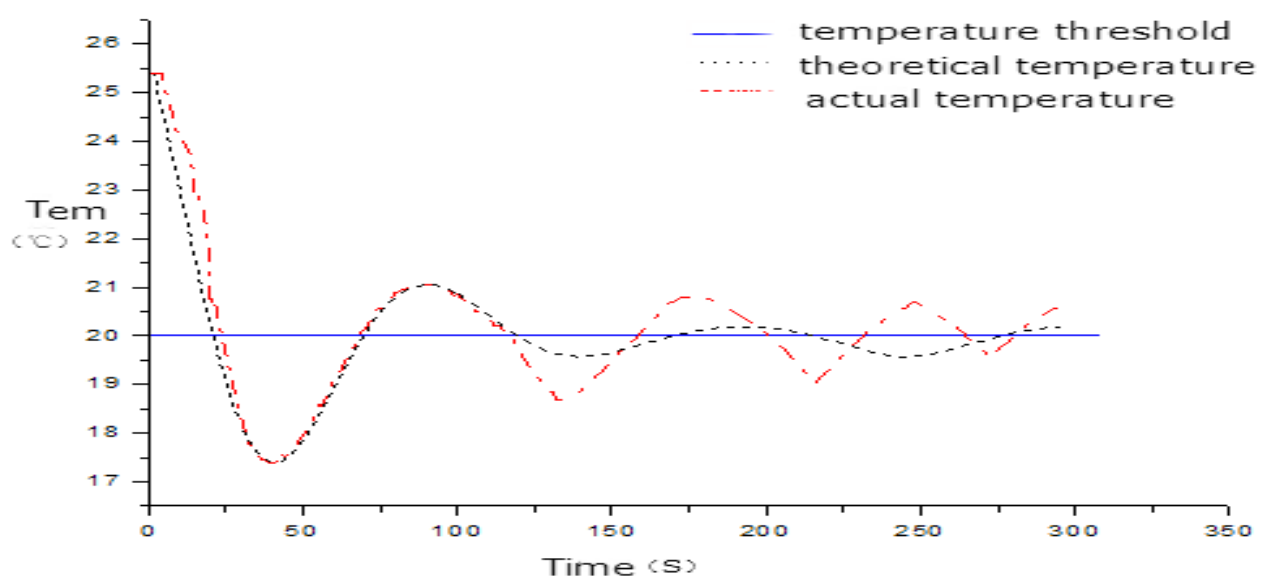

Figure 4 Diagram of temperature controlling effect

The collected data of constant temperature system threshold below room temperature is as table 1.

Table 1 Thermostatic system threshold below room temperature data collection form

\begin{tabular}{llll}
\hline Time & Left of box & Middle of box & Right of box \\
\hline 0min & $25.2^{\circ} \mathrm{C}$ & $25.4^{\circ} \mathrm{C}$ & $25.4^{\circ} \mathrm{C}$ \\
5 min & $21.3^{\circ} \mathrm{C}$ & $20.6^{\circ} \mathrm{C}$ & $20.9^{\circ} \mathrm{C}$ \\
$10 \mathrm{~min}$ & $19.8^{\circ} \mathrm{C}$ & $20.1^{\circ} \mathrm{C}$ & $19.6^{\circ} \mathrm{C}$ \\
$15 \mathrm{~min}$ & $20.3^{\circ} \mathrm{C}$ & $19.8^{\circ} \mathrm{C}$ & $20.1^{\circ} \mathrm{C}$ \\
\hline
\end{tabular}

In figure 3, the red curve represents the actual temperature, the yellow curve represents the 
theoretical temperature, the blue curve represents the set temperature threshold. The data of Table 1 is collected at the different parts of the column oven temperature on a fixed time interval ,by this set of data can provide data support for the precise control of various parts of the column oven . variation tendency from the red curve show the actual temperature drop is divided into stages and temperature stabilization phase, after 225 seconds the system enter into the temperature stabilization phase. under the control of the incremental PID algorithm, the value of a small area of the temperature and the temperature of the theory has a good agreement, because of the exchange principle of the temperature of the nature result that the actual temperature are some errors in the data, but in the end the system could be stabilized, error is within a controllable range .by the ratio of the critical ratio method tuning PID proportion P, the integral I, differential D parameters, ideal set of data is debugged within surplus overshoot, it will be saw that the overshoot of the actual temperature curve is reduced to $17.5 \%$.this control process reduces the overshoot and maintain a constant temperature system efficiently and quickly.

\section{Summary}

Small regional integrated climate control system is made up of a dual data collection, synchronous dual refrigeration heating control systems and incremental PID control algorithm. The algorithm combined with semiconductor temperature controller provides a set of high-precision temperature control system. solutions can effectively reduce outside interference, maintaining the temperature of the entire area of constant temperature changes in real-time monitoring system. this system temperature control effect is obvious, the structure has small size, and is suitable for most stringent temperature requirements systems, as well as the instrument cooling system, can effectively improve the instrument of practical life.

\section{Acknowledgments}

Thanks to the teacher's guidance and let me join the related projects include science and technology plan projects in Zhejiang province (2015C33009), science and technology plan projects in Jiaxing (2015 AY11008)

\section{References}

[1] Wang Hongjie,Du Jialian,Chen Jincan,Optimization on the Performa-nce Characteristics of a Semiconductor Refrigeration System, J. R-efrigeration, 1999,18（4） : 54-58.

[2]Fan Hanbai,Xie hanhua,Semiconductor Refrigerator Temperature Con-trol System with High-precision Based on Thyristor Phase-shifted Control, J. Instrument Technique and Sensor, 2012,5: 103-105.

[3] Cai Jinping,Li Li,The Small Area Temperature Control Model Based on Improved PID Algorithm Simulat, J. Computer Simulation,2015,32(6) : 237-240.

[4] Ge Leijiao,Mao Yizhi,Li Qi et al,RS232 Serial Interface Communic-ation with the C Language, J. Journal of Hebei University of Tech-nology,2008,37 (6) : 11-16.

[5] Xiao Wenjian,Li yongke,Design of Intelligent Vehicle Based on In-cremental PID Control Algorithm, J. Information technology, 2012, 10: 125-127.

[6] Yan Xiaozhao,Zhang Xingguo,Application of Increasing PID Contro-lling Method in Temperature Controlling System, J. Journal of Nan-tong University, 2006,5（4）: 48-51.

[7] Wang Shuyan, Shi Yu, Feng Zhongxu et al ,A Method for Controlling a Loading System Based on a Fuzzy PID Controller, J. Mechanical S-cience and Technology for Aerospace Engineering, 2011,30 (1) : 166-169. 
[8] LI Fengman., The Research of Controlling Arithmetic for Figure PID, J. Journal of Liaoning University, 2005,32 (4) : 367-370. 